\title{
Compréhension sociologique et démarches typologiques
}

Jacques Coenen-Huther

\section{OpenEdition}

1 Journals

Édition électronique

URL : https://journals.openedition.org/ress/272

DOI : $10.4000 /$ ress. 272

ISSN : 1663-4446

Éditeur

Librairie Droz

Édition imprimée

Date de publication : 1 août 2006

Pagination : 195-205

ISBN : 9-782-600-01108-2

ISSN : 0048-8046

Référence électronique

Jacques Coenen-Huther, "Compréhension sociologique et démarches typologiques », Revue européenne des sciences sociales [En ligne], XLIV-135 | 2006, mis en ligne le 13 octobre 2009, consulté le 21 septembre 2021. URL : http://journals.openedition.org/ress/272 ; DOI : https://doi.org/10.4000/ ress. 272

(c) Librairie Droz 


\section{COMPRÉHENSION SOCIOLOGIQUE ET DÉMARCHES TYPOLOGIQUES}

On admet de plus en plus généralement qu'un processus d'interprétation fondé sur la compréhension d'actions individuelles est une phase essentielle de toute analyse sociologique. Il importe en effet d'ancrer l'explication ou le diagnostic sociologique dans des séquences comportementales plausibles. C'est la plausibilité d'un enchaînement d'actions individuelles qui seule peut conférer un sens à la mise en relation de grandeurs agrégées. En ce sens, comme le note Dominique Schnapper, la compréhension sociologique remplace la confusion du réel «par un ensemble intelligible, cohérent et rationnel» $(2005, \text { p. } 1)^{1}$. La plausibilité d'une séquence comportementale s'impose avec la force de l'évidence lorsque la logique de l'acteur devient accessible au chercheur, non que celui-ci admette nécessairement le bien-fondé de la conduite adoptée ni qu'il en accepte les justifications mais qu'il puisse au moins en imaginer le déroulement sans éprouver un sentiment d'incohérence ou d'absurdité.

\section{LA COMPRÉHENSION, INDISPENSABLE MAIS PROBLÉMATIQUE}

L'opération de reconstruction de sens que nous appelons «compréhension» présuppose qu'une attitude de décentrement combinée à une recherche adéquate d'informations pertinentes doit permettre au sociologue de surmonter la distance sociale ou culturelle, voire même la distance historique, pour se situer mentalement dans une logique d'action et dans un contexte situationnel parfois très éloignés de ce qu'il peut être a priori tenté de considérer implicitement comme allant de soi. Ceci implique un pari sur l'existence de mécanismes fondamentaux de la vie en société et d'invariants du comportement humain permettant au chercheur de comprendre les mobiles de l'acteur par projection de raisons d'agir qui transcendent les époques et les cultures. Ce pari porte en fait sur les conditions de mise en œuvre de l'intention généralisante de la sociologie. De cette façon, il met en cause l'existence même de la sociologie comme projet scientifique. L'insistance sur la nécessité d'une phase de compréhension ne doit donc pas conduire à une pratique peu rigoureuse de la recherche sociologique, débouchant sur l'essayisme; elle est intimement liée au contraire à une «intention de science» qui ne

1 Et Dominique Schnapper a raison d'insister sur le caractère fondamental de la compréhension sociologique, allant bien au-delà de la mise en œuvre d'une prétendue «méthode compréhensive» (Schnapper, 2005, pp. 1-8). 
se satisfait pas de relations entre variables ne pouvant faire l'objet d'une attribution de sens. Les programmes de recherche où l'on cherche naïvement à éviter l'étape de compréhension par une mise en relation non sélective de variables ne donnent généralement pas de résultats concluants et plongent parfois les chercheurs dans la confusion. Les outils informatiques dont nous disposons actuellement permettent très facilement de croiser «tout avec tout» mais il n'est pas de plus sûr moyen de se rendre ridicule.

Si l'opération mentale de compréhension par effort d'identification à autrui apparaît comme un moment obligé de toute recherche, on doit pourtant bien reconnaître qu'elle reste un des maillons faibles de l'analyse sociologique ${ }^{2}$. Elle repose au moins en partie sur l'application d'une généralisation basée sur notre propre expérience ou sur notre sens personnel de l'évidence (Abel, 1948, p. 213). En dépit de l'ascèse intellectuelle la plus rigoureuse, le risque est toujours présent d'attribuer à l'acteur une logique de comportement dont on juge la portée universelle alors qu'elle est liée à un contexte socio-culturel particulier. Certes, il ne manque pas d'exemples construits qui suggèrent une compréhension aisée de la subjectivité de l'acteur. Gageons que l'attitude du piéton regardant à gauche et à droite avant de traverser la rue conservera encore quelque temps son pouvoir de fascination sur certains théoriciens. Mais il ne manque pas non plus d'exemples d'interprétations qui reflètent la subjectivité du chercheur bien davantage que celle de l'acteur. Dès qu'on s'écarte du banal élémentaire, on ne peut qu'être frappé par la résistance de la plupart des actions humaines non seulement à la compréhension explicative mais aussi à la compréhension la plus immédiate, celle que Max Weber qualifiait d'aktuelles Verstehen, fondée sur la perception directe de sens ou sur l'observation directe d'actions (1922, 1925, Vol. 1, pp. 3-4). Comme le souligne Raymond Aron dans son commentaire du point de vue weberien en la matière, l'intelligibilité intrinsèque a presque toujours pour contrepartie l'équivoque (Aron, 1967, 1991, pp. 504-505). Ceci implique le refus de l'intuition non contrôlée comme principe de compréhension. L'intuition ne pourrait être un support de compréhension suffisant que si tout acte directement observable était immédiatement compréhensible (Coenen-Huther, 1996, p. 139).

Pour éviter la projection abusive du point de vue du chercheur tout comme l'adhésion directe à celui de l'acteur, la relation de compréhension exige un optimum d'identité et d'altérité que seul peut assurer un va-et-vient sans cesse renouvelé entre la recherche de terrain et l'élaboration théorique ou conceptuelle. Après l'absorption d'informations nouvelles, la retraite intellectuelle est sans conteste la condition de la prise de distance nécessaire. Mais l'immersion périodique dans une réalité peu familière entretient la sensibilité comparative. La compréhension d'un comportement ne peut que s'appuyer sur quelque expérience antérieure qui le rende intelligible. Dès lors, les limites de l'expérience accumulée dictent les limites de la compréhension possible (Coenen-Huther, 1995, p. 175). C'est lorsqu'il est placé en contexte de découverte que le chercheur de terrain prend conscience de la manière la plus vive du caractère localisé de sa propre expérience

2 Je me permets de renvoyer à ce sujet à mon article «Formes et ambiguïtés de la compréhension en situation d'observation» dans lequel je m'appuie sur des expériences de terrain (Coenen-Huther, 1998, pp. 77-96). 
ou, si l'on préfère, du caractère soudain inadéquat de son propre « univers de référence $»^{3}$. C'est alors également qu'il est en mesure de s'affranchir du «sociocentrisme» (Piaget, 1965, p. 81) qui guette l'intellectuel coupé des stimulations de la recherche empirique et d'élargir ses possibilités de compréhension.

\section{L'ÉVIDENCE ET L'INTUITION}

Il n'en reste pas moins que le sentiment d'évidence n'assure le caractère convaincant d'une interprétation que s'il est largement partagé. L'intuition du chercheur individuel, pour tous ceux qui n'en ont pas été saisis de la même façon, «n'est qu'un fait» dont on ne peut que prendre acte (Dupréel, 1949, p. 76). Pour conférer à l'évidence un caractère transsubjectif, il faut donc que la démarche compréhensive se donne des normes de nature à discipliner le raisonnement et la récolte des données. Ceci est d'autant plus impérieux qu'on s'éloigne du type de recherche pouvant être soumis aux procédures de vérification standardisées. Un exemple d'évidence contestée nous est fourni par la recherche en milieu estudiantin publiée par Bourdieu et Passeron sous le titre Les héritiers (1964). Ces auteurs notent qu' «il semble légitime de voir dans les facultés des lettres le terrain par excellence pour étudier l'action des facteurs culturels de l'inégalité devant l'Ecole» (ibid., p. 21). Dominique Schnapper, approuvant la logique de la démarche adoptée dans cette enquête, y voit un exemple d'approche idéal-typique tenant «les étudiants en lettres pour les représentants les plus proches de l'idéaltype des étudiants du début des années 1960 » ( Schnapper, 2005, p. 26). Peu sensible à cette argumentation, Louis Gruel critique vigoureusement la procédure adoptée en se plaçant clairement dans une autre perspective. Il écrit en effet: "l'enquête de référence a été réalisée sans normes d'échantillonnage, auprès de quelques centaines d'étudiants exclusivement inscrits en faculté des lettres et sciences humaines » (2005, p. 20). Ce qui est ainsi remis en question est la représentativité du groupe d'étudiants soumis à l'enquête. Le commentaire critique se refuse à conférer au caractère typique le même degré d'évidence qu'à la représentativité statistique.

Il n'est donc pas superflu de s'interroger sur les fondements possibles du sentiment d'évidence que peut susciter une interprétation, lui offrant ainsi le statut de connaissance nécessaire. Il y a tout d'abord la «mathématisation» des données de l'expérience, dont on attend qu'elle aboutisse à des lois régissant les relations entre les faits quantifiés ${ }^{4}$. Il y a ensuite la référence à une forme de rationalité instrumentale, pouvant être reconnue par rapport au but visé, ou de rationalité axiologique ne s'imposant que par rapport à une échelle de valeurs. On attribue traditionnellement le niveau d'évidence supérieur à l'évidence mathématique dans la mesure où celle-ci se fonde commodément sur des critères formels et dispense de faire appel à des perceptions ou des représentations. Pourtant, même dans ce cas,

3 Pour reprendre les termes du regretté Jean-Michel Berthelot dans l'analyse d'une de mes expériences de terrain (Berthelot, 1996, pp. 229-231).

4 J'emprunte cette expression à Sylvie Mesure et Alain Renaut (Cf. La guerre des dieux. Essai sur la querelle des valeurs, 1996, pp. 79-80). 
le niveau d'évidence n'a pas une stabilité assurée; il arrive que le sentiment d'évidence suscité par le raisonnement mathématique fléchisse. Comme le fait observer Dupréel, lorsqu'on attribue à la formule $(\mathrm{a}+\mathrm{b}=\mathrm{c})$ la valeur $(2+2=4)$, «évidence et nécessité paraissent confondues dans une seule et même intuition ». Si en revanche on substitue aux termes $\mathrm{a}+\mathrm{b}$, des quantités complexes, «la dissociation de l'évidence et de la nécessité se produit» (1949, p. 84). Quoi qu'il en soit, nombreux sont les sociologues qui s'en tiennent volontairement aux objets de recherche se prêtant le plus facilement au traitement mathématique. La compréhension n'est pas nécessairement exclue du processus mais son importance s'en trouve relativisée. Il y a un demi-siècle, Theodore Abel suggérait qu'il y aurait lieu de tenir pour acquise une relation mise en évidence par des techniques statistiques appropriées, même si cette relation ne pouvait faire l'objet d'une attribution de sens (1948, p. 217). Les conceptions ont certes évolué depuis lors. De nos jours, peu de chercheurs accepteraient cette idée sans une certaine hésitation car un sentiment d'inquiétude les pousserait sans doute à suspendre leur jugement. Néanmoins, si l'attitude compréhensive et la quantification ne sont pas logiquement antithétiques, elles contribuent, dans la pratique, à définir des styles de recherche différents. La logique strictement causale associée à la quantification participe de l'adhésion à une épistémologie continuiste se refusant à établir une différence nette entre les sciences humaines et les sciences de la nature auxquelles on se réfère pour établir des critères de scientificité.

\section{L'IMPÉRATIF DE RÉDUCTION DE COMPLEXITÉ}

Cette attitude de subordination mentale aux sciences de la nature est-elle justifiée? La question n'en finit pas de préoccuper les sociologues. D'un point de vue très général, certains procédés de raisonnement peuvent être tenus pour communs aux deux groupes de disciplines, d'autant plus qu'ils ne font que prolonger des opérations élémentaires du sens commun. Dès qu'un être humain applique son esprit à l'examen d'une question quelconque, il se met plus ou moins consciemment à classer et à comparer. Et c'est ce qui se pratique tant en sociologie qu'en botanique. Comme le rappelle Dominique Schnapper «nous avons depuis Aristote, une tradition d'intelligibilité par la classification: répartir les choses - individus ou situations - selon un ou plusieurs critères » (2005, p. 15). Bernard Valade précise que c'est à l'époque des Lumières que la classification «s'est trouvée codifiée et son emploi généralisé comme mode d'explication scientifique par réduction de la diversité» (Valade, 2003, pp. 123-124). Depuis lors, tout projet scientifique s'appuie sur des opérations de classement et de comparaison, toute stratégie comparative implique le préalable de la classification. A ce niveau de généralité, il y a bien identité de méthode et les exposés de méthodologie sociologique renforcent souvent le sentiment d'unité de la démarche scientifique en se référant aux sciences de la nature et en traitant les termes «typologie» et «classification» comme des synonymes. Alain Degenne note que «l'usage consacre indifféremment typologie, classification, taxonomie, voire nomenclature, même si certains auteurs cherchent à réserver chaque terme à une méthode particulière » (2005, p. 713). Didier Demazière et Claude Dubar, traitant de typologies, y voient «une des opérations les plus courantes et les plus pratiquées dans les sciences 
sociales comme dans les sciences expérimentales ». Il s'agit selon eux, dans les premières comme dans les secondes, de «mettre de l'ordre dans les matériaux recueillis, les classer selon des critères pertinents, trouver les variables cachées qui expliquent les variations des diverses dimensions observables » (1997, p. 274). Pour Boudon et Bourricaud, la seule chose qui distingue une typologie d'une classification est le nombre de critères utilisés pour distinguer les éléments d'un ensemble. Lorsque la répartition se base sur un critère unique, on parle de classification; lorsqu'on utilise plusieurs critères, il peut être question de typologie. Il en résulte que «les typologies ne sont qu'une forme particulière de classification» (1982, p. 578). Dans le domaine des sciences sociales comme dans celui des sciences de la nature, le désir de «mettre de l'ordre» entraîne un souci de réduction de complexité qui est apparente dans les classifications de Buffon ou de Linné mais qu'on retrouve chez Durkheim. Dans le chapitre IV des Règles, celuici, s’inspirant fortement des procédés des sciences de la nature, recommande de substituer «à la multiplicité indéfinie des individus un nombre restreint de types » $(1895,1992, \text { p. } 79)^{5}$. Lorsqu'on examine de plus près cette idée de réduction de complexité, on s'aperçoit cependant qu'elle prend des formes différentes dans les sciences de la nature et dans les sciences humaines, en particulier en sociologie. Dans les sciences de la nature, quels que soient les critères adoptés, on s'en tient au principe aristotélicien de classement par le genre prochain et la différence spécifique. En sociologie, la réduction de complexité s'appuie sur des éléments d'appréciation extérieurs au principe même du classement.

\section{DURKHEIM, WEBER ET LA SÉLECTIVITÉ}

Pour Durkheim, le type social est fondé sur des faits «cruciaux » ou «décisifs » qui lui confèrent un aspect privilégié dans l'analyse. Ce qui définit ce caractère «crucial» ou «décisif», c'est son aptitude à s'insérer dans une classification des «espèces sociales» basée sur les principes taxinomiques des sciences naturelles. La mise en œuvre d'une morphologie sociale inspirée de la classification des espèces est le procédé qui semblerait permettre le recours à un principe de sélectivité interne au corpus de théorie sociologique en voie d'élaboration. Durkheim est néanmoins très attaché à opérer une distinction entre le normal et le pathologique. La typologie, dans son œuvre, a clairement pour objet d'introduire dans l'étude des faits sociaux une distinction entre le normal et l'anormal en évitant de conférer à ces termes une dimension normative. Il s'impose donc de faire usage de cette distinction tout en la relativisant. Dès lors, le type social constitue le niveau intermédiaire entre l'humanité prise globalement et chaque société en particulier. Il permet de relativiser la normalité tout en lui attribuant une signification pouvant être qualifiée de statistique; c'est la fréquence d'apparition d'un phénomène dans une société donnée qui définit son caractère normal ou anormal. Le normal est ainsi défini en dehors de toute valorisation consciente. Les faits cruciaux - ces instruments de réduction de complexité - servent à caractériser un type de sociétés

5 J'ai développé ceci dans mon article «Durkheim et l' impératif de réduction de complexité», in: Ch-H. Cuin, Ed., Durkheim d' un siècle à l'autre, Paris, PUF, 1997a, pp. 119-130. 
mais servent aussi à définir le normal et le pathologique. La pensée morale rejoint ici le projet scientifique et l'on s'écarte qu'on le veuille ou non de la pensée nomologique (Coenen-Huther, 1997a, pp. 121 et ss).

Si Durkheim, tout en s'inspirant de la classification des espèces naturelles, introduit un élément d'ambiguïté dans la typologie des espèces sociales, Max Weber s'écarte plus encore du principe de classement aristotélicien par l'accentuation délibérée du type idéal. Comme le note Dominique Schnapper, ce mode de conceptualisation correspond à une volonté de «stylisation de la réalité » (2005, p. 22). En l'adoptant à la suite de Montesquieu et de Tocqueville, Weber opte pour ce qu'on appelle de nos jours une logique de modélisation (Coenen-Huther, 2003, pp. 540 et ss). Le type idéal, comme le modèle, ne décrit pas la réalité empirique mais reflète un construit mental. Celui-ci n'entretient une relation avec le monde réel que moyennant un certain nombre de conditions qui doivent être spécifiées dans chaque cas. La congruence avec des faits empiriquement observables est du domaine du possible mais ce sont les écarts constatés entre la réalité et le type idéal qui ont les vertus heuristiques décisives. L'accentuation unilatérale produite par la conceptualisation idéal-typique résulte de la priorité accordée à l'un ou l'autre aspect de l'objet considéré. Cet élément de sélectivité consciente dépend d'une décision du chercheur et confère inévitablement un caractère historiquement daté à l'analyse. C'est la nature de l'objet - faits naturels ou événements historiques - qui est à la base du contraste entre les catégories de classification immuables des sciences de la nature et la démarche typologique du sociologue.

La différence entre les procédés de classement des sciences de la nature et ceux des sciences sociales a été bien perçue par Dominique Schnapper et par JeanClaude Passeron. Ceux-ci en concluent qu'il est préférable d'inscrire cette différence dans la terminologie en réservant le terme de typologie aux classifications des sciences sociales. Il est possible de se rallier à cette convention de langage en se rappelant néanmoins qu'elle est loin d'être acceptée par tous les sociologues. Mais en cela comme en d'autres matières, les concepts comptent beaucoup plus que les mots qui servent à les désigner. Dominique Schnapper note à ce sujet que « contrairement à la classification, l'élaboration d'une typologie ne consiste pas à regrouper des personnes ou des situations sociales sous un critère particulier mais à élaborer des relations abstraites entre les hommes ou les situations sociales; elle en révèle le trait essentiel qui permet de rendre intelligibles les formes que prennent ces relations ou ces situations» (2003, p. 306). Jean-Claude Passeron voit dans la classification et la typologie le résultat de deux méthodes distinctes. Pour lui, la classification résulte d'un «naturalisme inductiviste » inspiré du modèle des sciences de la nature alors que la typologie est le fruit d'une méthodologie idéaltypique liée «à la forme de présentation des phénomènes dans l'observation historique» (Passeron, 1994, pp. 7-8).

\section{LA SOCIOLOGIE, DISCIPLINE HYBRIDE}

Passeron présente Pareto et Weber comme les deux figures emblématiques de ces deux conceptions opposées des procédés classificatoires. Le premier, d'orientation positiviste, s'en remet à une «classification générique » à laquelle seule une «représentation nomologique des généralités historiques » peut conférer un potentiel 
opératoire. Le second, inspiré par une théorie de la connaissance d'inspiration néo-kantienne, développe une méthodologie idéal-typique en réaction aux illusions d'une «épistémologie mimétique des sciences de la nature» (ibid., pp. 6-9). Et Passeron marque fortement sa préférence pour la sociologie de type weberien en indiquant que lorsque l'analyse sociologique se fonde sur l'observation historique, elle présente ses énoncés «dans un langage dont les assertions ne doivent leur généralité conceptuelle qu'à leur forme typologique» (ibid., p. 5; souligné par JCH). Cette prise de position weberienne ${ }^{6}$ repose sur une conception de la sociologie comme discipline hybride, oscillant entre un pôle historique et un pôle expérimental (1990 et 1991) et c'est à cette conception qu'adhère Dominique Schnapper dans son plaidoyer pour la compréhension sociologique (2005, p. 136). Une discipline est historique, estime Passeron, lorsque les propositions qu'elle énonce ne peuvent être décontextualisées; en d'autres termes, lorsqu'elles ne peuvent être séparées «des contextes dans lesquels sont prélevées les données ayant du sens pour elle» (1990, p. 15). Le raisonnement sociologique présente souvent ce caractère historique mais il se distingue du raisonnement purement historique par «des moments de raisonnement expérimental» alternant avec des phases de «raisonnement naturel». C'est un raisonnement «mixte» qui ne peut que se déplacer entre les deux pôles dans la mesure où il se fonde sur «des faits datés et localisés » mais s'efforce d'échapper à «cette datation et cette localisation » en raison de sa visée généralisante (ibid. pp. 15-16). La volonté de généralisation du sociologue ne peut s'appuyer que sur des procédures fragiles au regard de la méthode expérimentale. Et si la stratégie comparative propre à la sociologie peut malgré tout prendre une dimension transculturelle ou transhistorique c'est grâce à la construction de types idéaux. Que l'on songe, par exemple, à l'opposition aristocratie-démocratie dans l'œuvre de Tocqueville. Lorsque celui-ci cherche une approximation historique du type aristocratique pur, il se tourne vers la France du XIe siècle. Cherchant alors la meilleure approximation du type démocratique, il le trouve dans les Etats-Unis de son temps ${ }^{7}$. Seul un mode de conceptualisation idéal-typique rend possible une stratégie comparative basée sur un tel voyage dans le temps et dans l'espace.

On comprend donc que toute recherche sociologique aux prises avec la tension entre recherche de sens et visée généralisante réserve une place de choix à la typologie dans ses procédés d'analyse. Comme d'autres, Dominique Schnapper a été confrontée à ces impératifs dans ses propres travaux. Analysant le rapport au judaïsme des juifs de la diaspora dans des sociétés fondées sur la citoyenneté, elle met en évidence trois modes de réinterprétation du judaïsme dans un contexte moderne: la «réinterprétation métaphysique» qui voit dans le judaïsme une croyance d'ordre religieux, la «réinterprétation historique» selon laquelle le judaïsme est lié à l'histoire collective d'un peuple, enfin la réinterprétation en termes de «destin collectif». (Schnapper 1980, 1993 et 2005). Ces réinterprétations sont des types idéaux et ces trois types idéaux forment une typologie des

6 Passeron indique qu'il aurait très bien pu appeler « weberien » l'espace du raisonnement sociologique qu'il a qualifié de «non-poppérien» (1994, p. 5, note 3).

7 J'ai présenté ceci plus en détail dans mon Tocqueville (1997b, chap. III, « Tocqueville, comparatiste avisé », pp. 52-78). 
réinterprétations possibles du judaïsme; aucun de ces types ne se retrouve à l'état pur «dans la réalité des expériences vécues» (2005, pp. 70-73).

\section{L'IMPOSSIBLE UNANIMITÉ}

Il faut bien voir toutefois que cette conception de la typologie commune à Dominique Schnapper et à Jean-Claude Passeron est une conception particulière, liée à une certaine conception de la sociologie et dont on peut supposer qu'elle ne fera jamais l'unanimité des chercheurs de terrain. Nos auteurs envisagent en effet une démarche typologique strictement liée au type idéal comme outil conceptuel. Ils sont ainsi tous deux amenés à utiliser le terme «typologie» comme synonyme de méthode idéal-typique. Ceci requiert à nouveau une convention d'ordre terminologique car les typologies fondées sur des types idéaux ne constituent qu'un cas particulier parmi les approches typologiques utilisées en sociologie. Grémy et Le Moan (1977), dont Dominique Schnapper connaît bien les travaux, proposent de distinguer trois démarches typologiques différentes, la méthode idéal-typique n'étant que l'une d'entre elles. Outre celle-ci, ces auteurs distinguent la méthode de «la réduction de l'espace d'attributs» et la méthode de «l'agrégation autour d'unités-noyaux ».

La méthode de la réduction de l'espace d'attributs a été commentée en détail par Lazarsfeld (1993, pp. 158-168). Celui-ci rappelle que la notion de «type» renvoie à une certaine combinaison d'attributs. Dans un «espace d'attributs », plusieurs attributs sont pris en considération et ceci peut être visualisé de façon comparable à un cadre de référence en géométrie analytique. Chaque attribut - par exemple, la taille, la beauté ou le niveau de formation - peut être placé sur un axe et les différents axes peuvent être croisés pour obtenir des combinaisons variées (ibid., p. 159). L'analyse part d'une combinatoire des dimensions logiquement possibles pour aboutir, par réduction et élimination, à une typologie fondée sur la distribution empiriquement observable. L'opération ne se résume cependant pas, comme le suggèrent Demazière et Dubar, à une manœuvre à caractère technique ayant pour but «d'éliminer les cases vides - ou presque vides - et de ne retenir que celles où se trouvent un nombre significatif d'éléments » (1997, p. 275). En fait, des hypothèses plus ou moins explicitement formulées entrent en jeu. L'exemple ci-dessous, emprunté à Lazarsfeld, le fera aisément comprendre ${ }^{8}$.

Soit un espace d'attributs où interviennent trois caractéristiques: avoir ou ne pas avoir un diplôme d'études supérieures, être un Blanc ou un Noir, être né aux Etats-Unis ou être né à l'étranger. Il en résulte huit combinaisons dont, par exemple, la combinaison «Blanc - né à l'étranger - sans diplôme d'études supérieures». Supposons qu'on veuille estimer l'avantage (ou le désavantage) social lié aux différentes combinaisons. On pourra éventuellement raisonner sur le désavantage fondamental que subissent les Noirs aux Etats-Unis; les différentes combinaisons incluant le fait d'être un Noir pourront dès lors être regroupées en une seule pour constituer une catégorie de «désavantage maximum». A la suite

8 L'exemple est fictif et les raisonnements qu'il implique ne sont pas nécessairement confirmés par les faits. 
d'autres raisonnements impliquant les deux autres caractéristiques, un ordre d'avantage social décroissant est constitué, à savoir 1) Blanc, né aux Etats-Unis, possédant un diplôme d'études supérieures; 2) Blanc, né aux Etats-Unis, sans diplôme; 3) Blanc, né à l'étranger, avec ou sans diplôme; 4) Noir, quel que soit le lieu de naissance ou le niveau de formation. Les huit combinaisons de départ sont ainsi ramenées à quatre: on a donc réduit l'espace d'attributs (Lazarsfeld, 1993, pp. 159-160). C'est alors qu'intervient l'analyse statistique.

Comme on peut le constater, on ne se trouve pas ici face à des types idéaux. Les exemples évoqués par Lazarsfeld portent sur des groupes d'individus pouvant faire l'objet de classements en fonction de divers critères. Il n'en reste pas moins qu'un élément de compréhension est partie intégrante de la procédure. Le regroupement des catégories ne s'opère pas de façon mécanique; il doit faire sens pour le chercheur. Ce regroupement est fondé sur des hypothèses qui viennent à l'esprit de manière plus ou moins intuitive. Seule une connaissance de la société considérée permet de les envisager et cette connaissance est préalable au traitement statistique des données. La procédure inclut une phase de compréhension sociologique qui est masquée par la quantification ultérieure mais qui n'en est pas moins présente.

Contrairement aux deux autres démarches, la méthode de l'agrégation autour d'unités-noyaux, appelée également «méthode des tas», est fondée sur une procédure inductive. Elle ne doit cependant rien au modèle paretien des sciences de la nature. Le renouveau des sociologies interactionnistes d'une part, le succès de la stratégie d'ancrage de l'élaboration théorique dans la recherche empirique - la stratégie dite de la grounded theory - d'autre part, ont suscité un regain de confiance dans les approches inductives qui permettent l'élaboration graduelle d'un cadre conceptuel et d'une problématique de recherche à mesure que progresse le travail de terrain. Bien des réticences subsistent à l'égard des stratégies inductives qui n'offrent pas le confort mental de la «construction d'objet» préalable». Mais le nombre de chercheurs qui se placent résolument dans une optique de découverte plutôt que dans une optique prudente de vérification augmente.

Dans la «méthode des tas», les données dont on dispose sur chaque unité d'observation - individu, groupe ou situation d'interaction - sont rassemblées dans des dossiers et condensées sur des fiches maniables. Les données font l'objet d'un classement donnant lieu à une répartition provisoire des fiches à partir d'unités-noyaux. C'est la «mise en tas» des dossiers individuels. Les réexamens successifs des fiches - avec retour éventuel aux dossiers de base - aboutissent à un affinement progressif de la classification. Concrètement, il peut être question de création de nouveaux tas, de division d'un tas existant, ou encore de fusion de tas. Au cours de cette procédure by trial and error, les critères de classification se précisent ${ }^{9}$. On aboutit ainsi à des types qui ne sont pas des types idéaux au sens weberien mais qui n'en permettent pas moins de mettre en évidence des individus, des discours ou des situations typiques, pouvant avoir un effet structurant ultérieur. Pour Demazière et Dubar, la réussite d'une telle procédure inductive exige un «non-savoir théorique» au départ. Le chercheur, précisent-ils, «doit se dire

9 C'est une procédure de ce genre que Josette Coenen-Huther a mis en œuvre dans sa recherche sur les types de mémoire familiale (La mémoire familiale, 1994). 
qu'il ne sait rien» (1997, p. 277). Hormis le fait que certains chercheurs n'éprouveront guère de difficultés à se conformer à cette injonction, on ne peut que voir dans cet empirisme radical une conception inquiétante des relations entre la recherche et la théorie: inquiétante parce qu'elle n'est guère favorable à la constitution d'un savoir cumulatif en sociologie. Elle n'y est pas plus favorable que certaines prises de position empiristes de Glaser et Strauss dont les travaux tout à fait remarquables ne gagnent rien à se couper volontairement de toute élaboration théorique préalable.

\section{POUR CONCLURE}

Un élément de compréhension est présent dans toute analyse proprement sociologique: on en sera d'accord avec Dominique Schnapper. On souscrira également à sa proposition selon laquelle la typologie est le procédé de classification le plus adapté à la démarche sociologique. On sera néanmoins tenté de séparer analytiquement compréhension, typologie et approche idéal-typique. Sans doute, une typologie fondée sur des types idéaux est difficilement concevable en dehors d'une posture méthodologique compréhensive. En revanche, l'attribution de sens que vise la compréhension peut surgir en dehors de toute démarche typologique. Quant à la typologie, elle est fréquemment liée à une approche idéal-typique mais elle peut également reposer sur des types construits d'une autre manière. Ces distinctions mettent en cause le caractère hybride de la sociologie qui apparaît dans l'oscillation entre son pôle historique et son pôle expérimental. Si plusieurs procédés de construction typologique coexistent, c'est précisément en raison de cet écartèlement qui traverse toute l'entreprise sociologique. Qu'il s'agisse de la procédure déductive de la réduction de l'espace d'attributs ou de la procédure inductive de l'agrégation autour d'unités-noyaux, on se situe ici plus près du pôle expérimental que du pôle historique de la sociologie. Ces procédures sont valablement utilisées dans de nombreuses recherches empiriques dont la dimension historique est absente, non par carence mais parce que la longue durée est absente de leur problématique. L'option entre logique inductive et logique déductive est alors plus pertinente que l'option entre classification à caractère typologique et classification d'inspiration nomologique.

\section{Carouge/Genève}

\section{RÉFÉRENCES BIBLIOGRAPHIQUES}

Abel, Theodore, «The Operation called Verstehen», The American Journal of Sociology, Vol. 54, 1948-1949, pp. 211-218.

Aron, Raymond, Les étapes de la pensée sociologique, Paris, Gallimard, 1967, réédition «Tel», 1991.

Berthelot, Jean-Michel, Les vertus de l'incertitude, Paris, PUF, Coll. «Sociologie d'aujourd'hui », 1996.

Boudon, Raymond et Bourricaud, François, Dictionnaire critique de la sociologie, Paris, PUF, 1982.

Bourdieu, Pierre et Passeron, Jean-Claude, Les héritiers. Les étudiants et la culture, Paris, Minuit, 1964. 
Coenen-Huther, Jacques, Observation participante et théorie sociologique, Paris, L'Harmattan, 1995.

Coenen-Huther, Jacques, «L'observation participante et l'épistémologie piagétienne», Revue Européenne des Sciences Sociales, Tome XXXIV, No 106, 1996, pp. 133-140.

Coenen-Huther, Jacques, «Durkheim et l'impératif de réduction de complexité». In: Charles-Henry Cuin, Ed., Durkheim d'un siècle à l'autre. Lectures actuelles des Règles de la méthode sociologique, Paris, PUF, 1997a, pp. 119-130.

Coenen-Huther, Jacques, Tocqueville, Paris, PUF, Coll. «Que sais-je?», 1997b.

Coenen-Huther, Jacques, «Formes et ambiguïtés de la compréhension en situation d'observation», Cahiers internationaux de Sociologie, Vol. CIV, 1998, pp. 77-96.

Coenen-Huther, Jacques, «Le type idéal comme instrument de la recherche sociologique», Revue française de sociologie, 44-3, juillet-septembre 2003, pp. 531-547.

Coenen-Huther, Josette, La mémoire familiale. Un travail de reconstruction du passé, Paris, L'Harmattan, 1994.

Degenne, Alain, «Typologie», in: Borlandi, Massimo et al., Dictionnaire de la pensée sociologique, Paris, PUF, 2005, pp. 713-717.

Demazière, Didier et Dubar, Claude, Analyser les entretiens biographiques. L'exemple des récits d'insertion, Paris, Nathan, 1997.

Dupréel, Eugène, Essais pluralistes, Paris, PUF, 1949.

Durkheim, Emile, Les règles de la méthode sociologique, Paris, 1895, PUF, Quadrige, 1992.

Grémy, Jean-Pierre et Le Moan, Marie-Joëlle, «Analyse de la démarche de construction de typologies dans les sciences sociales », Informatique et sciences humaines, No 35, 1977.

Gruel, Louis, Bourdieu illusionniste, Rennes, Presses Universitaires de Rennes, 2005.

Lazarsfeld, Paul, «Classifying and Building Typologies », On Social Research and its Language, Chicago et Londres, The University of Chicago Press, 1993

Mesure, Sylvie et Renaut, Alain, La guerre des dieux. Essai sur la querelle des valeurs, Paris, Grasset, 1996.

Passeron, Jean-Claude, «L'enseignement, lieu de rencontre entre historiens et sociologues », débat avec Antoine Prost, Sociétés contemporaines, No 1, mars 1990, pp. 7-45.

Passeron, Jean-Claude, Le raisonnement sociologique: l'espace non-poppérien du raisonnement naturel, Paris, Nathan, 1991.

Passeron, Jean-Claude, «La rationalité et les types de l'action sociale chez Max Weber», Revue Européenne des Sciences Sociales, Tome XXXII, No 98, 1994, pp. 5-44.

Piaget, Jean, Etudes sociologiques, Genève, Droz, 1965.

Schnapper, Dominique, Juifs et israélites, Paris, Gallimard, Coll. «Idées », 1980.

Schnapper, Dominique, «Le sens de l'ethnico-religieux », Archives des sciences sociales des religions, Vol. 81, 1993, pp. 149-163.

Schnapper, Dominique, «L'analyse typologique », in: Moscovici, Serge et Buschini, Fabrice, Eds., Les méthodes des sciences humaines, Paris, PUF, «Fondamental», 2003, pp. 297-314.

Schnapper, Dominique, La compréhension sociologique. Démarche de l'analyse typologique, édition revue et augmentée, Paris, PUF, Quadrige, 2005.

Valade, Bernard, «De la classification et des classificateurs», in: Cherkaoui, Mohamed, Ed., Histoire et théorie des sciences sociales. Mélanges en l'honneur de Giovanni Busino, Genève, 2003, pp. 123-135.

Weber, Max, Wirtschaft und Gesellschaft, 1922, $2^{\mathrm{e}}$ édition augmentée, publiée comme $3^{\mathrm{e}}$ section de l'ouvrage collectif Grundriss der Sozialökonomik, Tubingen, J.C.B. Mohr (Paul Siebeck), 2 vol., 1925 . 